\title{
POLYCYCLIC AROMATIC HYDROCARBON AND MID-INFRARED CONTINUUM EMISSION IN A $z>4$ SUBMILLIMETER GALAXY
}

\author{
Dominik A. Riechers ${ }^{1,2}$, Alexandra Pope ${ }^{3}$, Emanuele Daddi $^{4}$, Lee Armus $^{5}$,

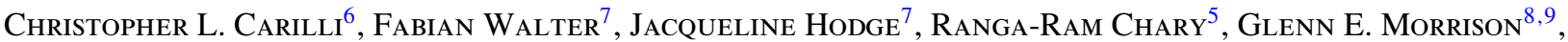 \\ Mark Dickinson $^{10}$, Helmut Dannerbauer $^{11}$, And David Elbaz ${ }^{4}$ \\ ${ }^{1}$ Department of Astronomy, Cornell University, 220 Space Sciences Building, Ithaca, NY 14853, USA; dr@astro.cornell.edu \\ ${ }^{2}$ Astronomy Department, California Institute of Technology, MC 249-17, 1200 East California Boulevard, Pasadena, CA 91125, USA \\ ${ }^{3}$ Department of Astronomy, University of Massachusetts, Amherst, MA 01003, USA \\ ${ }^{4}$ Laboratoire AIM, CEA/DSM-CNRS-Université Paris Diderot, Irfu/Service d'Astrophysique, CEA Saclay, \\ Orme des Merisiers, F-91191 Gif-sur-Yvette Cedex, France \\ ${ }^{5}$ Spitzer Science Center, California Institute of Technology, MC 220-6, 1200 East California Boulevard, Pasadena, CA 91125, USA \\ ${ }^{6}$ National Radio Astronomy Observatory, PO Box O, Socorro, NM 87801, USA \\ ${ }^{7}$ Max-Planck-Institut für Astronomie, Königstuhl 17, D-69117 Heidelberg, Germany \\ ${ }^{8}$ Canada-France-Hawaii Telescope, 65-1238 Mamalahoa Hwy, Kamuela, HI 96743-8432, USA \\ ${ }^{9}$ Institute for Astronomy, 2680 Woodlawn Drive, Honolulu, HI 96822-1839, USA \\ ${ }^{10}$ National Optical Astronomy Observatory, 950 North Cherry Avenue, Tucson, AZ 85719, USA \\ ${ }^{11}$ Institut für Astrophysik, Universität Wien, Türkenschanzstraße 17, A-1180 Wien, Austria \\ Received 2013 June 21; accepted 2014 March 10; published 2014 April 14
}

\begin{abstract}
We report the detection of $6.2 \mu \mathrm{m}$ polycyclic aromatic hydrocarbon (PAH) and rest-frame $4-7 \mu \mathrm{m}$ continuum emission in the $z=4.055$ submillimeter galaxy GN20, using the Infrared Spectrograph on board the Spitzer Space Telescope. This represents the first detection of PAH emission at $z>4$. The strength of the PAH emission feature is consistent with a very high star formation rate of $\sim 1600 M_{\odot} \mathrm{yr}^{-1}$. We find that this intense starburst powers at least $\sim 1 / 3$ of the faint underlying $6 \mu \mathrm{m}$ continuum emission, with an additional, significant (and perhaps dominant) contribution due to a power-law-like hot dust source, which we interpret to likely be a faint, dust-obscured active galactic nucleus (AGN). The inferred $6 \mu \mathrm{m}$ AGN continuum luminosity is consistent with a sensitive upper limit on the hard X-ray emission as measured by the Chandra X-Ray Observatory if the previously undetected AGN is Compton-thick. This is in agreement with the finding at optical/infrared wavelengths that the galaxy and its nucleus are heavily dust-obscured. Despite the strong power-law component enhancing the mid-infrared continuum emission, the intense starburst associated with the photon-dominated regions that give rise to the PAH emission appears to dominate the total energy output in the infrared. GN20 is one of the most luminous starburst galaxies known at any redshift, embedded in a rich protocluster of star-forming galaxies. This investigation provides an improved understanding of the energy sources that power such exceptional systems, which represent the extreme end of massive galaxy formation at early cosmic times.
\end{abstract}

Key words: cosmology: observations - galaxies: active - galaxies: formation - galaxies: high-redshift - galaxies: starburst - infrared: galaxies

Online-only material: color figures

\section{INTRODUCTION}

Submillimeter galaxies (SMGs; see review by Blain et al. 2002) are thought to be the progenitors of the most massive present-day galaxies, making them a key ingredient to studies of massive galaxy formation and evolution through cosmic time. Many SMGs are outliers on the stellar-mass-star-formation-rate relation at $z \sim 2$ (e.g., Daddi et al. 2007), and they typically host intense ( $>500-1000 M_{\odot} \mathrm{yr}^{-1}$ ) starbursts of a few kiloparsecs in diameter, embedded in $\gtrsim 10 \mathrm{kpc}$ gas reservoirs (e.g., Riechers et al. 2011a, 2011b; Ivison et al. 2011). They frequently feature rapid gas consumption through high star formation efficiencies that are associated with ongoing major mergers (e.g., Tacconi et al. 2008). The high abundance of passive massive galaxies already by $z \sim 2$ (e.g., Renzini 2006) requires a substantial population of $z>3-4$ galaxies with vigorous starbursts. Studies of the so-called high-redshift tail of SMGs at $z>4$ thus promise to provide valuable insight toward understanding the distribution of formation redshifts of massive early-type galaxies. The few $z>4$ SMGs securely identified to date (e.g., Daddi et al. 2009a [hereafter: D09], 2009b; Capak et al. 2008, 2011; Riechers et al.
2010, 2013) may already be sufficient to account for known populations of old massive galaxies at $z=2-3$ (e.g., Coppin et al. 2009). Also, $z>4$ SMGs may significantly contribute to the contemporaneous comoving star formation rate density (e.g., D09).

GN20 $(z=4.055)$ is one of the intrinsically brightest SMGs known $\left(S_{850 \mu \mathrm{m}}=20.3 \mathrm{mJy}\right.$; Pope et al. 2005,2006$)$ and one of only a few spectroscopically confirmed unlensed SMGs at $z>4$. Located in a massive galaxy protocluster structure $\left(\sim 10^{14} M_{\odot}\right.$; D09; Hodge et al. 2013), this remarkable galaxy hosts a massive $M_{\mathrm{H}_{2}}=1.8 \times 10^{11}\left(\alpha_{\mathrm{CO}} / 1.1\right) M_{\odot},{ }^{12} 14 \pm 4 \mathrm{kpc}$ diameter molecular gas disk, which accounts for $\sim 1 / 3$ of the total (dynamical) mass of the galaxy (Hodge et al. 2012 [H12]). This gas reservoir feeds an intense starburst that is almost entirely obscured by dust at optical wavelengths within the central $\gtrsim 10 \mathrm{kpc}$ (e.g., Iono et al. 2006; Carilli et al. 2010 [C10]). This intense, $>1000 M_{\odot} \mathrm{yr}^{-1}$ starburst will be sufficient to turn GN20 into one of the most massive galaxies known within

$12 \alpha_{\mathrm{CO}}$ is the conversion factor from CO luminosity to molecular gas mass (H12). 
$\ll 1$ Gyr. Due to the high level of obscuration, it is currently not known if GN20 hosts an active galactic nucleus (AGN), and thus, if the infrared luminosity $\left(L_{\mathrm{IR}}\right)$ used to estimate the star formation rate is entirely powered by dust-reprocessed light from young stars.

At low and intermediate redshifts $(z \sim 2)$, spectral decomposition of mid-infrared spectra has proven to be a powerful tool to investigate the relative contributions of AGNs and star formation to the infrared light in star-forming galaxies (e.g., Armus et al. 2007; Farrah et al. 2007; Pope et al. 2008 [P08]; Veilleux et al. 2009; Petric et al. 2011), but no constraints exist for galaxies at $z>4$ so far.

Here, we report the detection of $6.2 \mu \mathrm{m}$ polycyclic aromatic hydrocarbon (PAH) emission and 4-7 $\mu \mathrm{m}$ continuum emission toward the $z=4.055$ SMG GN20. This is the highest $z$ detection of PAH emission reported to date, reaching back to only 1.5 Gyr after the Big Bang. This enables us to better constrain the intensity of the starburst in this galaxy and to determine the presence and energy contribution of an AGN in this intriguing system. We use a concordance, flat $\Lambda \mathrm{CDM}$ cosmology throughout, with $H_{0}=71 \mathrm{~km} \mathrm{~s}^{-1} \mathrm{Mpc}^{-1}$, $\Omega_{\mathrm{M}}=0.27$, and $\Omega_{\Lambda}=0.73$ (Spergel et al. 2003, 2007).

\section{OBSERVATIONS}

Observations toward GN20 $(z=4.055)$ were carried out with the Infrared Spectrograph (IRS; Houck et al. 2004) on board the Spitzer Space Telescope on 2009 May 2-4 with a total observing time of $24 \mathrm{hr}$, split up into $120 \mathrm{~s}$ integrations (DDT project 531; PI: D. Riechers). As the $6.22 \mu \mathrm{m}$ PAH feature in GN20 is redshifted to $31.44 \mu \mathrm{m}$, we selected the first order of the Long-Low module (LL1; 19.5-38.0 $\mu \mathrm{m}$ ) for this study. To improve the calibration accuracy, these ultra-deep observations were obtained in spectral mapping mode, placing the target at six different positions (separated by $20^{\prime \prime}$ ) along the slit. High-accuracy peak-ups on isolated, bright Two Micron All Sky Survey (2MASS) stars were obtained in the blue filter to optimize the pointing of the telescope.

The basic calibrated data (BCD) files produced by the Spitzer IRS S18.7.0 pipeline (including ramp fitting, dark sky subtraction, droop and linearity corrections, flat-fielding, and wavelength calibration) were used for further calibration and processing. The two-dimensional dispersed frames were corrected for rogue pixels (using IRSCLEAN), after which a small percentage of the remaining bad pixels was rejected. Then, the data were corrected for latent charge buildup (dominantly from zodiacal background), which was fitted by a constant polynomial and a time-variable component as a function of wavelength and then subtracted. The sky background was removed by fitting and subtracting a normalized "supersky" map (in which all sources on the slit are masked) created from the data at different slit positions within an astronomical observation request (AOR; see P08 for details), resulting in minimal residual sky noise. After sky removal, individual data frames were co-added using a clipped median and cleaned once more for persisting hot pixels. The Spitzer IRS Custom Extraction (SPICE) program was then used to "blindly" extract one-dimensional spectra from the two-dimensional coadds for each nod position at the known $24 \mu \mathrm{m}$ position of the (unresolved) source (which is too faint to be detected in individual two-dimensional coadds). The uncertainty in the one-dimensional spectra was estimated from extracting residual sky spectra in the off-source position of each two-dimensional coadd. The final spectrum was obtained by averaging over all nod positions. The flux scale was obtained in SPICE by extracting spectra of standard calibrator stars obtained during the same campaign as our science observations and found to be in good agreement with existing $24 \mu \mathrm{m}$ photometry of the source. The final noise on the spectrum between 21 and $32.5 \mu \mathrm{m}$ is estimated to be $40-90 \mu \mathrm{Jy}$ per $0.169 \mu \mathrm{m}$ wavelength bin. The spectrum was trimmed above observed-frame wavelengths of $\sim 35 \mu \mathrm{m}$ due to high noise. The nearby SMG GN20.2 was not covered by these observations due to the IRS slit orientation at the time of observation.

\section{RESULTS}

We have detected $6.2 \mu \mathrm{m}$ PAH emission and 4-7 $\mu \mathrm{m}$ continuum emission toward the $z=4.055$ SMG GN20 (Figure 1 left panel). The PAH emission extends over at least five wavelength bins and is detected at $>6 \sigma$ significance (when simultaneously fitting the underlying continuum). The continuum is detected at typically $1.5 \sigma-4 \sigma$ per wavelength bin over the full spectral range ( $>80$ bins), allowing us to constrain the continuum slope over this spectral range. The strength of the continuum emission is consistent with the $24 \mu \mathrm{m}$ flux of $65.5 \pm 3.5 \mu \mathrm{Jy}$ measured with the Multiband Imaging Photometer for Spitzer (MIPS; P08; D09) within the (considerable) relative uncertainties. When accounting for the steeply sloped continuum emission, the PAH line shape is symmetric and consistent with a Gaussian or Lorentzian profile, as expected. A Gaussian fit to the line profile and nearby continuum (i.e., the same method as used by P08) yields an integrated $6.2 \mu \mathrm{m}$ PAH luminosity of $L_{\mathrm{PAH}(6.2 \mu \mathrm{m})}=(2.70 \pm 0.44) \times 10^{10} L_{\odot}$, and a rest-frame line equivalent width of $0.17 \pm 0.08 \mu \mathrm{m}$. A small positive excess is seen close to the expected peak of the $5.7 \mu \mathrm{m}$ PAH feature, but not enough to claim a detection. The spectrum also shows a tentative decrement consistent with the wavelength of the $4.67 \mu \mathrm{m} P$ and $R$ branches of the fundamental vibrational mode of $\mathrm{CO}$, which is observed in absorption toward some nearby ultra-luminous infrared galaxies with embedded nuclei (e.g., Spoon et al. 2004, 2005). Unfortunately, the sensitivity is not sufficient to claim a detection of this $\mathrm{CO}$ absorption feature.

We extract the remaining spectral properties of the source by fitting a two-component model to the data, consisting of an extincted power-law component (with a wavelength-dependent optical depth obtained from the Draine 2003 extinction curves) and a starburst component (see, e.g., Menéndez-Delmestre et al. 2007, 2009; P08). The starburst component is an M82 template (Förster Schreiber et al. 2003); however, adopting the starburst composite template of Brandl et al. (2006) yields the same results within the uncertainties. ${ }^{13}$ In comparison to the starburst template, the PAH feature is both narrow and has a low equivalent width. ${ }^{14}$ Based on the examination of subsets of the data, the narrow shape of the feature relative to templates is of limited significance. Low PAH equivalent widths, such as those observed for GN20, are typically found in galaxies with significant AGN emission at mid-infrared wavelengths

\footnotetext{
13 The $6.2 \mu \mathrm{m}$ PAH to $6 \mu \mathrm{m}$ continuum flux ratio among the starbursts that are part of this template vary by about $\pm 20 \%$, which may be considered the minimum level of uncertainty in the decomposition. This is due to the fact that hot dust emission from a very compact, obscured starburst can also cause some level of excess in the mid-infrared continuum emission of a galaxy.

14 The $6.2 \mu \mathrm{m}$ PAH equivalent width for the starburst template reported by Brandl et al. (2006) is $0.53 \mu \mathrm{m}$, with values in the range of $0.459-0.789 \mu \mathrm{m}$ for individual galaxies that are part of the template (measured using a method that gives similar results to our method; see the discussion by P08).
} 

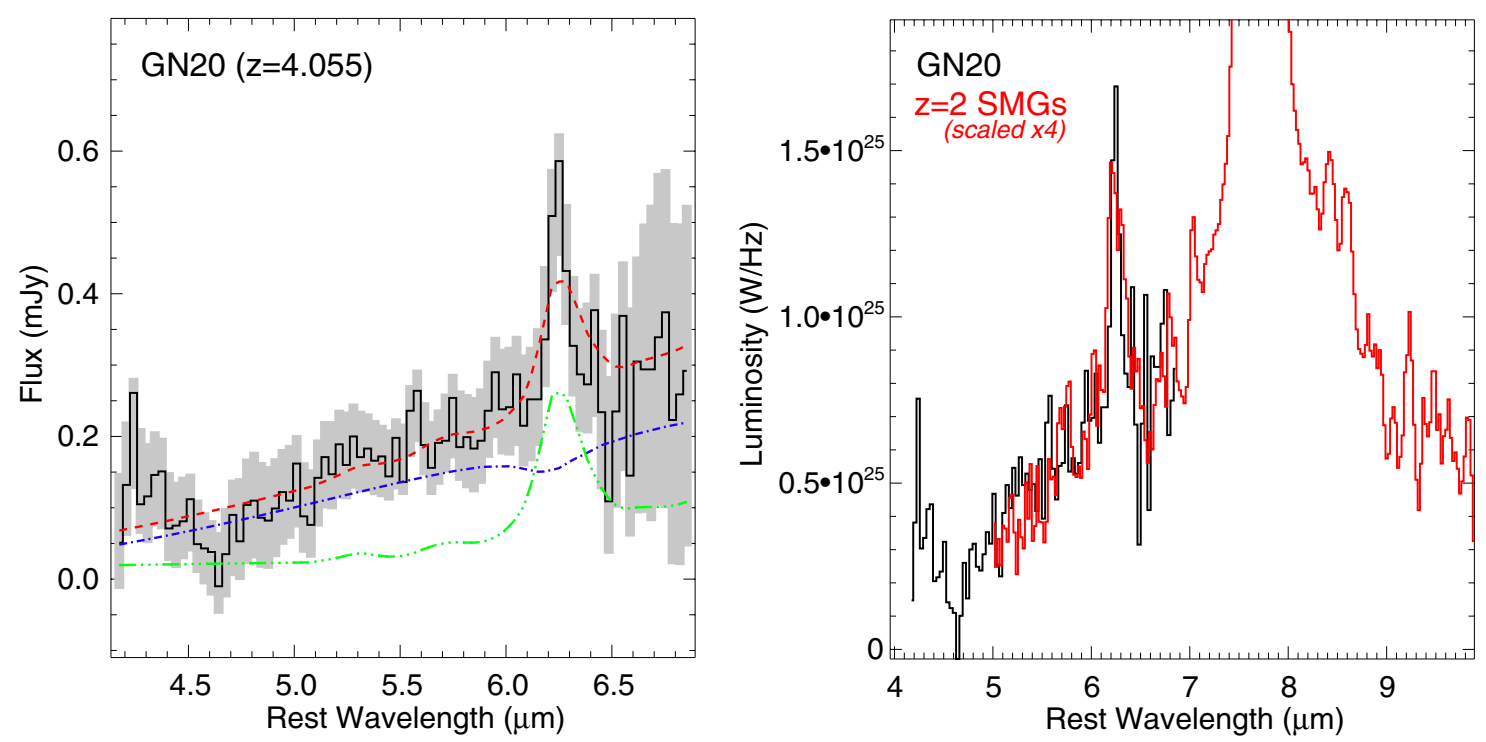

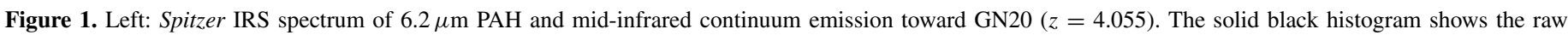

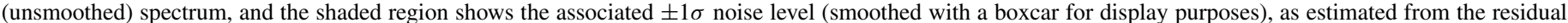

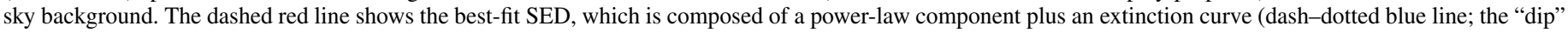

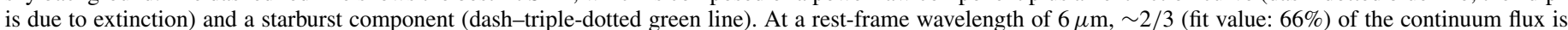

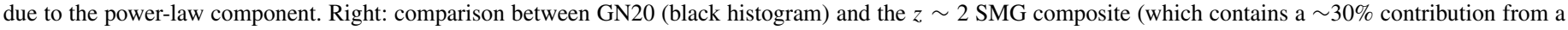
power-law AGN component) shown in Pope et al. (2008), scaled up by a factor of four in luminosity (red histogram).

(A color version of this figure is available in the online journal.)

(e.g., Armus et al. 2007). Within the limited spectral range, the spectral decomposition suggests that $\sim 2 / 3$ (fit value: $66 \%$ ) of the continuum emission at $6 \mu \mathrm{m}$ is due to the powerlaw component, $\sim 1 / 3(34 \%)$ contribution from the starburst template, and a level of uncertainty of at least $30 \%$ from the fitting alone. The power-law component alone, likely due to a hot dust source, corresponds to a rest-frame $6 \mu \mathrm{m}$ continuum luminosity of $\nu L_{\nu}(6 \mu \mathrm{m})=2.6 \times 10^{45} \mathrm{erg} \mathrm{s}^{-1}$. As discussed below, we interpret this power-law component to be mainly a result of a heavily dust-enshrouded AGN in GN20, tracing the thermally re-radiated emission from the obscuring material.

In the right panel of Figure 1, a comparison between the spectrum of GN20 and the $z \sim 2$ SMG composite spectrum of $\mathrm{P} 8^{15}$ (scaled up by a factor of four in luminosity) is shown. Except for a narrower PAH feature in GN20, both spectra look quite similar. The average AGN contribution for the $z \sim 2$ SMG composite sources is $\sim 30 \%$ (P08). This shows that the presence of an AGN in GN20 is likely, but that, due to the limited spectral range of our observations, the AGN contribution to the rest-frame $6 \mu \mathrm{m}$ continuum luminosity is probably uncertain by about a factor of two.

For comparison, we have extracted the rest-frame X-ray 2-10 keV luminosity of GN20 from the deep 2 Ms exposure of the region obtained with the Chandra X-Ray Observatory (Alexander et al. 2003). The source is not detected down to a limiting luminosity of $L_{\mathrm{X}}(2-10 \mathrm{keV})<1.8 \times 10^{42} \mathrm{erg} \mathrm{s}^{-1}$ (based on the observed-frame $0.5-2 \mathrm{keV}$ flux limit, assuming a $\mathrm{K}$ correction of 1.085 and a spectral slope of $\Gamma=1.4$ ). Under the assumption that GN20 hosts a mid-infrared-luminous, obscured AGN, this X-ray limit provides interesting constraints on its AGN properties (as outlined below).

\footnotetext{
15 The $6.2 \mu \mathrm{m}$ PAH equivalent widths for individual SMGs that are part of this template are $0.22-1.10 \mu \mathrm{m}$, which includes two upper limits of $<0.45$ and $<0.50 \mu \mathrm{m}(\mathrm{P} 08)$.
}

\section{ANALYSIS}

\subsection{Spectral Energy Distribution}

Our spectral decomposition suggests that $\sim 34 \%-70 \%$ of the mid-infrared continuum flux in GN20 is due to star formation. This suggests that, in order to determine the fraction of $L_{\mathrm{IR}}$ that is due to star formation, the photometry at these wavelengths needs to be corrected down accordingly to fit only the starburst fraction of the spectral energy distribution (SED). We have applied this correction to the SED fit presented by Magdis et al. (2011; conservatively adopting the 34\% value from the twocomponent fit). This yields a starburst infrared luminosity of $L_{\mathrm{IR}}=(1.9 \pm 0.4) \times 10^{13} L_{\odot}$, which is essentially the same as the total infrared luminosity quoted by Magdis et al. (2011). Given the SED shape of the source, the impact of the correction (and its factor of $\sim 2$ uncertainty) on the total $L_{\mathrm{IR}}$ is minor. The correction does not account for a potential cooler dust component that may be subject to AGN heating, but that cannot be described by the mid-infrared power-law component. At present, there is no evidence for the existence of such an additional component in GN20.

To better quantify the possible contribution of any additional, unconstrained component to $L_{\mathrm{IR}}$ if present, we simultaneously fit the mid-infrared spectrum and full infrared SED of GN20 with a composite full SED template of $z \sim 2$ SMGs (consisting of the same galaxies used in the mid-infrared composite in Figure 1; P08) and an SED template of the $z=0.042$ AGN-dominated infrared-luminous galaxy Mrk 231 (Figure 2; Rigopoulou et al. 1999; P08). The estimated contribution of star formation to the far-infrared luminosity of Mrk 231 is $30 \% \pm 15 \%$ (Veilleux et al. 2009). This simultaneous composite fit reproduces the overall SED properties of GN20 fairly well, and it suggests a starburst contribution of $35 \%$ to the mid-infrared luminosity. This agrees well with the estimate based on the decomposition of the mid-infrared spectrum alone. The fit overestimates the luminosity at $\sim 4.4-5.0 \mu \mathrm{m}$. This may be due to the possible 


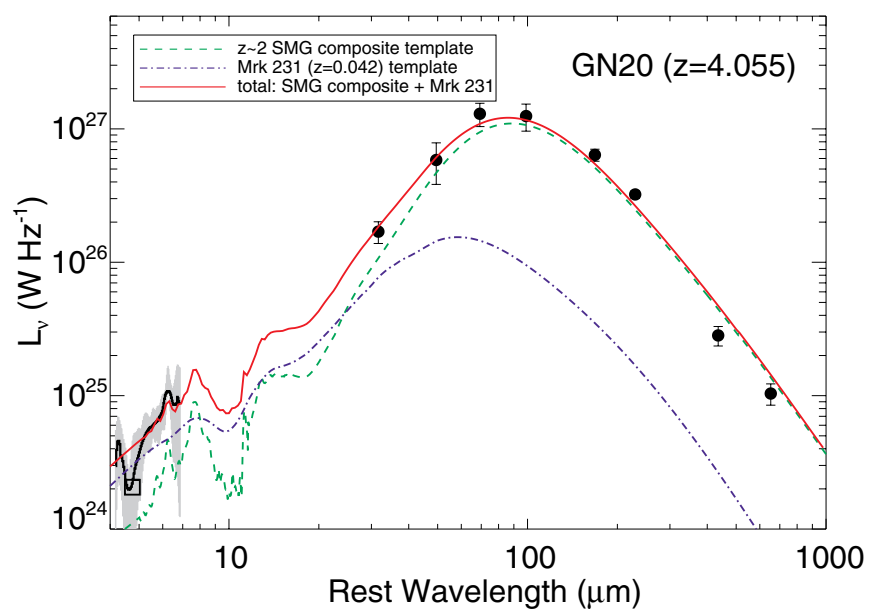

Figure 2. Spectral energy distribution of GN20 in specific luminosity $\left(L_{v}\right)$, overlaid with the Spitzer IRS spectrum and spectral templates. The IRS spectrum (thick black line and gray shaded area) was smoothed for clarity. The Spitzer MIPS $24 \mu \mathrm{m}$ photometry $(65.5 \pm 3.5 \mu \mathrm{Jy}$; open square; P08, D09) is shown for comparison. Rest-frame $>30 \mu \mathrm{m}$ photometry (filled circles) is adopted from Magdis et al. (2011). The error bars of the Herschel SPIRE photometry are corrected for a contribution due to confusion noise (e.g., Nguyen et al. 2010). The green dashed line shows a smoothed composite SED template for $z \sim 2$ SMGs (P08). The blue dash-dotted line shows an SED template of Mrk 231 (Rigopoulou et al. 1999; P08). The red long solid line shows a composite of both templates, fitted to the GN20 data by allowing a constant scaling factor for both components (i.e., $\mathrm{SED}_{\text {total }}=a_{1} \mathrm{SED}_{\mathrm{SMG}}+a_{2}$ SED Mrk231).

(A color version of this figure is available in the online journal.)

presence of $\mathrm{CO}$ absorption in this spectral region, but it could also indicate a lower AGN contribution to the mid-infrared luminosity, which is consistent with the range allowed by the mid-infrared spectral decomposition alone. The fit also suggests that the AGN contribution to the total $L_{\mathrm{IR}}$ is $\lesssim 15 \%-20 \%$. Given the underlying assumptions, we consider this an approximate upper limit for the AGN contribution to $L_{\mathrm{IR}}$ in the following.

We can compare our results to those for individual $z \sim 2$ SMGs. In the sample of P08, three $z \sim 2$ SMGs (classified as starbursts in the mid-infrared) have upper limits on the AGN continuum contribution to their mid-infrared spectra of $<29 \%$ to $<35 \%$, and three other $z \sim 2$ SMGs (classified as AGN+starburst systems in the mid-infrared) have estimates in the range of $47 \%-61 \%$. For these galaxies, which have mid-infrared AGN continuum fractions consistent with GN20, P08 find upper limits of $<15 \%$ to $<35 \%$ and estimates of $11 \%-32 \%$ for the AGN contribution to their total $L_{\text {IR }}$ of $0.2-1.0 \times 10^{13} L_{\odot}$. For five $z \sim 2.5 \mathrm{SMGs}$ selected to have a steeply rising continuum slope between 4.5 and $8 \mu \mathrm{m}$ (as expected for SMGs with a significant AGN contribution at those wavelengths), Coppin et al. (2010) find 26\%-62\% AGN contribution in the mid-infrared and $23 \%-36 \%$ AGN contribution to their total $L_{\mathrm{IR}}$ of $0.5-1.5 \times 10^{13} L_{\odot} \cdot{ }^{16}$ This suggests a conservative limit of $<1 / 3$ for a contribution of such an additional component to the $L_{\mathrm{IR}}$ in GN20, if present. This is consistent with the results from the composite SED fitting.

Based on the $1.4 \mathrm{GHz}$ radio flux density of $72 \pm 13 \mu \mathrm{Jy}(\mathrm{C} 10$; Pope et al. 2006; Morrison et al. 2010), we find a $q$ parameter (i.e., ratio of $L_{\mathrm{IR}}$ to monochromatic $1.4 \mathrm{GHz}$ radio luminosity; Helou et al. 1985) of $q=2.39$ for GN20 ( $q=2.32$ if assuming

\footnotetext{
16 Systems in the samples of P08 and Coppin et al. (2010) with mid-infrared AGN continuum contributions that are not consistent with GN20 were excluded from this comparison.
}

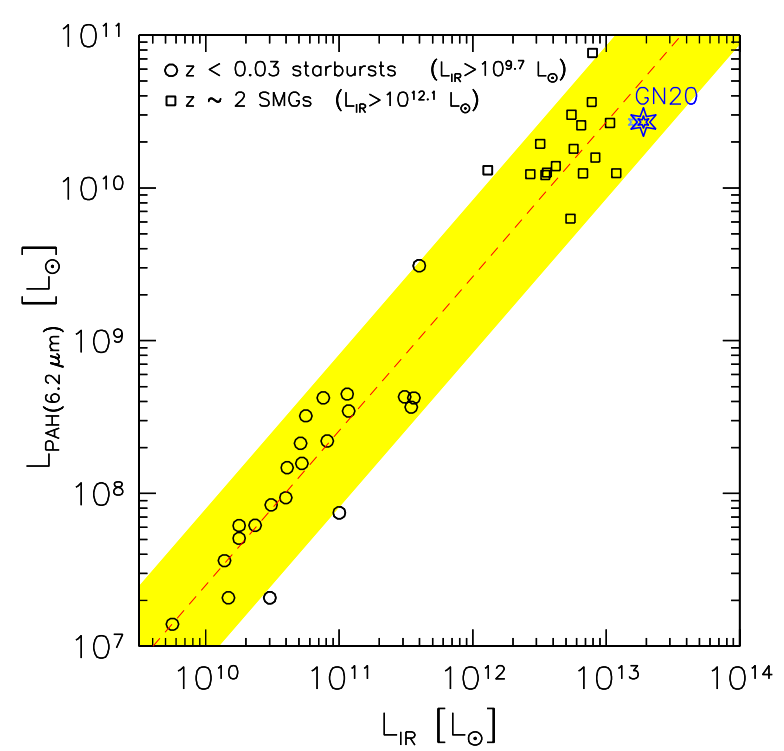

Figure 3. Relation between $L_{\mathrm{IR}}$ (corrected for AGN contribution) and the $6.2 \mu \mathrm{m}$ PAH luminosity, $L_{\mathrm{PAH}(6.2 \mu \mathrm{m})}$, for the local $(z<0.03)$ starburst galaxy sample from Brandl et al. (2006; circles), the $z \sim 2$ SMG sample from P08 and Coppin et al. (2010; squares), and the $z=4.055$ SMG GN20 (this work; stars). The line shows the best-fit relation found by P08: $\log L_{\mathrm{PAH}(6.2 \mu \mathrm{m})}=1.01 \log L_{\mathrm{IR}}-2.7$. The shaded region indicates a factor of three scatter. The small star shows the position of GN20 when assuming a $15 \%$ lower $L_{\mathrm{IR}}$.

(A color version of this figure is available in the online journal.)

an AGN contribution of $15 \%$ to $L_{\mathrm{IR}}$ ), which is consistent with galaxies that follow the radio-infrared correlation for nearby star-forming galaxies $(q=2.3 \pm 0.1$; Yun et al. 2001) and SMGs with solid radio identifications $(q=2.4 \pm 0.1$; Ivison et al. 2010). This is consistent with no significant AGN contribution to the $L_{\mathrm{IR}}$ in GN20 beyond the corrections employed above. There is also no evidence of an AGN contribution to the radio emission in GN20, in contrast to its nearby companion galaxy, GN20.2a, which hosts a radio-detected AGN (D09).

\subsection{AGN and Star Formation Relations}

Interstellar PAH particles typically occur in photondominated regions (PDRs), which are associated with starforming clouds. In these clouds, young stars heat large dust grains that emit reprocessed stellar light in the infrared. Consequently, a relation between $L_{\mathrm{PAH}(6.2 \mu \mathrm{m})}$ and $L_{\mathrm{IR}}$ was found over a range in luminosity from individual local star-forming regions up to starburst galaxies (e.g., Peeters et al. 2004) and even $z \sim 2$ SMGs (P08). In Figure 3, we plot this relation for nearby starburst galaxies (Brandl et al. 2006), the $z \sim 2$ SMG sample from P08 and Coppin et al. (2010), and GN20. All infrared luminosities are corrected for their estimated AGN contribution. The (linear) relation shows a scatter by a factor of $\sim 3$ (as indicated by the shaded region), likely due to differences in the physical properties of the individual star-forming environments in these galaxies (such as starburst geometry and clumpiness, dust distribution and temperature, or viewing angle toward a merger). The $z=4.055$ SMG GN20 follows this relation within the scatter, providing further evidence that its huge bolometric energy output is dominantly due to an intense starburst. Given its high $L_{\mathrm{IR}}$ relative to nearby starbursts, the relative strength of the PAH feature in GN20 is consistent with a fairly large spatial extent of the starburst, but relatively moderate dust temperatures (rather than a compact, hot starburst region), such that 


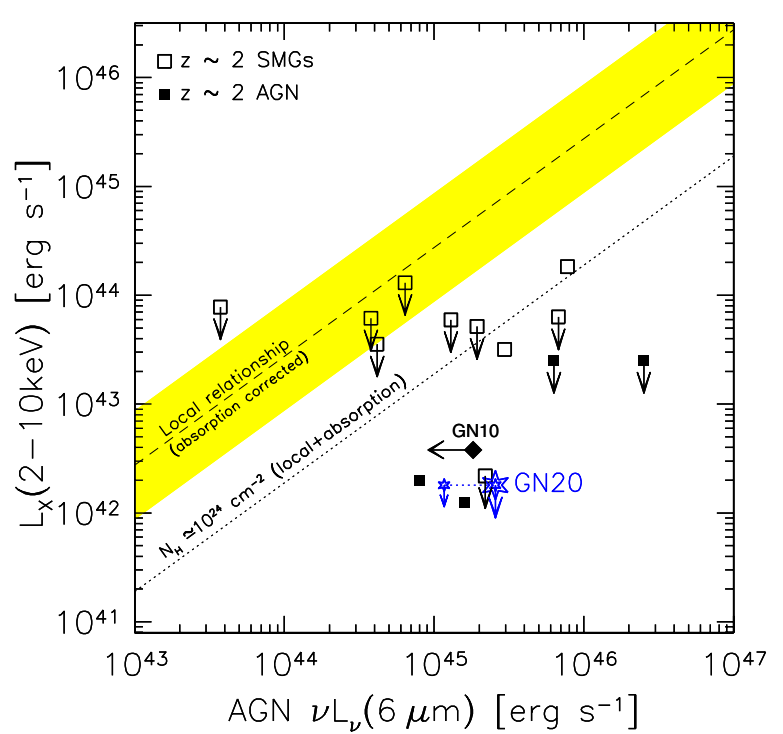

Figure 4. Relation between the rest-frame mid-IR $6 \mu \mathrm{m}$ continuum luminosity (power-law component only) and the rest-frame X-ray 2-10 keV luminosity for the $z \sim 2$ SMG sample from Coppin et al. (2010; empty squares), including C1 from P08, the $z \sim 2$ AGN sample from Alexander et al. (2008; filled squares), the $z=4.042$ SMG GN10 (Laird et al. 2010; Pope et al. 2006; an upper limit assuming $100 \%$ power-law contribution to $L_{v}(6 \mu \mathrm{m})$ and the same mid-infrared continuum slope as GN20 is shown; filled diamond), and the $z=4.055$ SMG GN20 (this work; stars; the large symbol indicates the value from the spectral decomposition, the small symbol indicates an assumed powerlaw contribution of $30 \%$ to $\left.L_{v}(6 \mu \mathrm{m})\right)$. The black dashed line and shaded region indicate the absorption-corrected relation for nearby AGNs (Lutz et al. 2004). For comparison, the black dotted line indicates the expected luminosity ratio for an absorbing column density of $N_{\mathrm{H}} \simeq 10^{24} \mathrm{~cm}^{-2}$, using the model by Alexander et al. (2005a). Assuming that the local relation holds, sources significantly below this line (which include GN20) may be considered Compton-thick.

(A color version of this figure is available in the online journal.)

typical PDRs giving rise to the PAH emission are largely intact. This is in agreement with the moderate molecular gas surface densities found for the bulk of the gas reservoir (H12) and with the moderate characteristic dust temperature found from SED fitting (33 K; Magdis et al. 2011). Younger et al. (2008) place a limit of $\sim 4-8 \mathrm{kpc}$ (depending on the assumed source morphology) on the FWHM diameter of the starburst in GN20, which is consistent with a fairly large total extent as expected from the relative PAH strength.

In nearby AGNs, hard X-ray emission from the central engine is commonly considered to be a measure of the bolometric AGN luminosity and thus should be linked to the strength of the thermally re-radiated mid-infrared emission from hot dust close to the nucleus. This finding has led to the discovery of an empirical relation between $L_{X}(2-10 \mathrm{keV})$ and $\nu L_{\nu}(6 \mu \mathrm{m})$ (e.g., Lutz et al. 2004), which is linear over three to five orders of magnitude and extends out to high redshift. Galaxies can significantly deviate from this relation due to intervening matter along the line of sight to the AGN, i.e., due to a significant absorbing column density of obscuring dust at X-ray wavelengths that lowers the observed X-ray fluxes. In Figure 4, the (absorption-corrected) relation for nearby AGNs is compared to a sample of $z \sim 2$ AGNs and SMGs (Alexander et al. 2008; Coppin et al. 2010), the $z=4.042$ SMG GN10 (Laird et al. 2010; Pope et al. 2006), and GN20. For all galaxies, only the estimated AGN power-law contribution to the mid-infrared emission from the spectral decomposition is shown (for GN10, only an upper limit exists due to the lack of mid-infrared spectroscopy). The deep X-ray limit indicates that GN20 would be obscured by a factor of $>430$ if the local relation were to hold (with a factor of at least $\sim 3$ uncertainty due to the scatter in that relation, as indicated by the shaded region in Figure 4, and some additional uncertainty in the K correction and X-ray spectral slope assumed for GN20). This suggests that if an AGN contributes at $\sim 30 \%-66 \%$ level to the mid-infrared continuum emission (as indicated by the spectral decomposition), it is likely a type-2 AGN obscured by Compton-thick material. This would be in agreement with its non-detection in the optical, and with the extreme obscuration of the massive, gas- and dust-rich host galaxy (e.g., D09; C10; H12). This AGN is unlikely to be very luminous; even when assuming a correction factor of $\gtrsim 430$, the limit on $L_{X}(2-10 \mathrm{keV})$ corresponds to only $\sim 1 \%$ of $L_{\mathrm{IR}}$, which is far below the values typically found for luminous AGNs (Alexander et al. 2005a).

\subsection{Star Formation Rate}

Based on the relation between $L_{\mathrm{PAH}(6.2 \mu \mathrm{m})}$ and $L_{\mathrm{IR}}, \mathrm{P} 08$ have determined a relation to convert $L_{\mathrm{PAH}(6.2 \mu \mathrm{m})}$ into a star formation rate (SFR; their Equation (7)). For GN20, this relation suggests an SFR of $\sim 1600 M_{\odot} \mathrm{yr}^{-1}$ when assuming a Chabrier (2003) stellar initial mass function (IMF). With the same IMF, the $L_{\mathrm{IR}}$ of GN20 suggests an SFR of $1900 M_{\odot} \mathrm{yr}^{-1}\left(\sim 1600 M_{\odot} \mathrm{yr}^{-1}\right.$ if assuming a $15 \%$ AGN contribution to $\left.L_{\mathrm{IR}}\right)$, comparable to that of the $z=5.3$ SMG AzTEC-3 (which also resides in a protocluster environment; Capak et al. 2011; Riechers et al. 2010). Therefore, these estimates consistently suggest an SFR of at least $\sim 1600 M_{\odot} \mathrm{yr}^{-1}$ within the relative uncertainties.

Given the large molecular gas mass of $1.8 \times 10^{11}\left(\alpha_{\mathrm{CO}} /\right.$ 1.1) $M_{\odot}(\mathrm{H} 12)$, this corresponds to a gas consumption timescale of $\sim 10^{8}$ yr. This is consistent with a short, intense burst of star formation, as seen in "typical" $z \sim 2$ SMGs (e.g., Greve et al. 2005) and $z \gtrsim 4$ quasar host galaxies (e.g., Riechers et al. 2008a, 2008b).

\section{DISCUSSION}

We have detected $6.2 \mu \mathrm{m}$ PAH and rest-frame $4-7 \mu \mathrm{m}$ continuum emission toward the $z=4.055$ SMG GN20 using the Spitzer IRS. GN20 is one of the most luminous dusty galaxies known at high redshift. The $6.2 \mu \mathrm{m}$ PAH equivalent width is a few times lower than in "typical" $z \sim 2$ SMGs due to a relatively high contribution $(\sim 1 / 3-2 / 3)$ of a power-law component to the continuum emission. When fit in the same fashion, $z \sim 2$ SMGs typically show $<30 \%$ contribution of power-law continuum emission in the mid-infrared ( $~ 80 \%$ of the population; e.g., P08).

The simplest compelling interpretation is that the different characteristics of GN20 relative to nearby starbursts and the majority of "typical" $z \sim 2$ SMGs are related to a luminous power source besides the starburst, with a possible contribution from other mechanisms and/or due to unusual properties of the dust. This additional, luminous power source is likely an obscured AGN. Given the lack of direct evidence for an AGN at other wavelengths, this interpretation warrants further exploration.

On the one hand, there is no known mechanism other than AGN activity that can produce the high excess in mid-infrared continuum luminosity that is observed in GN20 without making fairly extreme assumptions. The tentatively identified $4.67 \mu \mathrm{m}$ $\mathrm{CO}$ absorption feature, if confirmed, would be consistent with a very warm, embedded source contributing significantly to the $6 \mu \mathrm{m}$ continuum emission (against which the absorption 
is measured), such as an obscured AGN. In principle, a very compact, obscured starburst can cause some excess in the mid-infrared continuum emission of a galaxy, which in fact may be responsible for at least part of the variations seen among the mid-infrared spectra of nearby starbursts. However, GN20 is dominated by a moderately warm, spatially extended starburst, without evidence of a significant compact, hot subcomponent. It has been argued in the literature that small, hot dust grains in the most energetic $\mathrm{H}$ II regions can produce power-law-shaped radiation in the mid-infrared (e.g., Genzel et al. 1998; Tran et al. 2001). Due to the high redshift of GN20 and the likely presence of multiple emission components, the spectral coverage of the IRS spectrum is not sufficient to firmly distinguish between the flatter mid-infrared power-law slopes of emission associated with a dust torus surrounding an AGN and the steeper slopes observed toward $\mathrm{H}$ II regions with highly concentrated star formation. Given the overall cool dust SED shape of GN20, it remains unclear if such $\mathrm{H}$ II regions are sufficiently common to produce a power-law-shaped excess component as strong as observed. In any case, given a possible contribution from $\mathrm{H}$ II regions, we consider our estimates for the power-lawshaped component to be an upper limit for the actual AGN contribution.

On the other hand, there are several potential reasons for the low observed equivalent width of the PAH feature. One possible explanation of the low equivalent width of the PAH feature is that the adopted nearby starburst template (or even the $z \sim 2$ SMG template) may not be suitable to fit the spectrum of substantially more luminous and/or distant starburst galaxies at $z>4$. Besides the relative strength of the hot dust continuum emission, the equivalent width of the $6.2 \mu \mathrm{m}$ PAH feature depends on the ionized versus neutral PAH ratio, which in turn depends on metallicity and the hardness of the radiation field that the PAH particles are bathed in (e.g., Draine \& Li 2007). The relative strength of the $\mathrm{CO}$ emission in GN20 compared to its farinfrared dust properties (D09; C10; H12), however, does not indicate substantial differences compared to $z \sim 2$ SMGs (e.g., Tacconi et al. 2008; Riechers et al. 2011a, 2011b) in terms of dust/gas-phase metallicity. Furthermore, the overall radiation field that penetrates the dust would be substantially harder if composed of both a starburst and an AGN component. This could be consistent with a non-standard ionized versus neutral $\mathrm{PAH}$ ratio, which may result in a narrower feature and a lower equivalent width, as seen in GN20 (however, it may be difficult to reconcile a scenario where the AGN radiation penetrates the opaque, obscuring dust and gas throughout the large, $14 \mathrm{kpc}$ diameter of the reservoir).

Interestingly, the $z \sim 2 \mathrm{SMG}$ with the lowest equivalent width $6.2 \mu \mathrm{m}$ PAH feature $(\mathrm{C} 1,0.05 \pm 0.01 \mu \mathrm{m}$; i.e., $<1 / 3$ the equivalent width of GN20) found by P08, like GN20, is consistent with hosting a Compton-thick AGN. Studies of other types of galaxies find that systems with $6.2 \mu \mathrm{m}$ PAH equivalent widths of $<0.2 \mu \mathrm{m}$ are typically AGN-dominated in the mid-infrared (e.g., Armus et al. 2007; Sajina et al. 2007). Also, Coppin et al. (2010) have studied a sample of $z \sim 2$ SMGs that was suspected to be dominated by AGNs (representing $<15 \%$ of the $z \sim 2$ SMG population), ${ }^{17}$ several of which are at least consistent with being a Compton-thick AGN. These studies find that in a number of these systems (corresponding

\footnotetext{
17 With $S_{8 \mu \mathrm{m}} / S_{4.5 \mu \mathrm{m}}=2.75$, GN20 formally follows the color excess (caused by $500-1000 \mathrm{~K}$ hot dust) selection criterion for mid-infrared AGN-dominated SMGs as defined by Coppin et al., but only due to the shorter rest-frame wavelengths probed in these bands at $z>4$ relative to their $z \sim 2.5$ sample.
}

to $\lesssim 5 \%$ of the SMG population, not including the $\sim 4 \%$ of SMGs that are type-1 AGN/quasars), the AGN is bolometrically important, and sometimes even dominant, relative to their submillimeter-detected luminous starbursts of a few hundred to $>1000 M_{\odot} \mathrm{yr}^{-1}$. Our findings show that an obscured, Comptonthick AGN may significantly contribute to, and perhaps dominate, the energy output in the mid-infrared in GN20, but it appears to only yield a minor contribution to the bolometric energy output of the galaxy.

In conclusion, we have used the Spitzer IRS to constrain the AGN and star formation properties in a starburst galaxy at $z>4$, employing the unique diagnostics observable only at midinfrared wavelengths. Based on the detection of $6.2 \mu \mathrm{m}$ PAH and 4-7 $\mu \mathrm{m}$ continuum emission in the exceptional $z=4.055 \mathrm{SMG}$ GN20, we have found evidence for the likely presence of a heavily buried, Compton-thick, but not bolometrically dominant, AGN hosted by a massive, dust-obscured 1600-1900 $M_{\odot} \mathrm{yr}^{-1}$ starburst.

We thank Dave Alexander for help with obtaining the $\mathrm{X}$-ray limit and for communicating some data source tables, Henrik Spoon for helpful comments, Georgios Magdis for help with an earlier version of Figure 2, and the anonymous referee for a helpful and constructive report. D.R. acknowledges support from a Spitzer Space Telescope grant related to this project. D.R. appreciates the hospitality at the Aspen Center for Physics, where part of this manuscript was written. This work is based on observations made with the Spitzer Space Telescope, which is operated by the Jet Propulsion Laboratory, California Institute of Technology, under a contract with NASA. The IRS was a collaborative venture between Cornell University and Ball Aerospace Corporation funded by NASA through the Jet Propulsion Laboratory and Ames Research Center. The scientific results reported in this article are based in part on observations made by the Chandra X-ray Observatory.

\section{APPENDIX}

\section{A.1. Black Hole Mass and Accretion Rate}

Assuming that GN20 hosts an obscured AGN and that the local $v L_{v}(6 \mu \mathrm{m})-L_{X}(2-10 \mathrm{keV})$ relation holds, i.e., that the large absorption corrections found in Section 4.2 are valid, we can attempt to derive an Eddington limit for the black hole mass $M_{\mathrm{BH}}$ of GN20 based on $v L_{v}(6 \mu \mathrm{m})$. Assuming a bolometric correction of $\kappa_{2-10 \mathrm{keV}}=L_{\mathrm{bol}} / L_{\mathrm{X}}(2-10 \mathrm{keV})=55$ (for high $L_{\text {bol }} / L_{\text {edd }}$ galaxies; Vasudevan \& Fabian 2007), we find $M_{\mathrm{BH}}^{\mathrm{Edd}} \simeq 1.4-3.0 \times 10^{8} M_{\odot}$ for $30 \%-66 \%$ of $v L_{v}(6 \mu \mathrm{m})$, corresponding to $\sim 0.03 \%-0.06 \%$ of the total (dynamical) mass of the galaxy $\left(M_{\mathrm{dyn}}=5.4 \pm 2.4 \times 10^{11} M_{\odot} ; \mathrm{H} 12\right)$. This ratio is comparable to what is found in $z \sim 2$ SMGs $(\sim 0.05 \%$; Alexander et al. 2005b, 2008). This may imply that the $M_{\mathrm{BH}}$ of GN20 falls short of the near-linear local $M_{\mathrm{BH}}-M_{\text {bulge }}$ relation (which corresponds to a mass ratio of $0.19 \%$ at the $M_{\text {dyn }}$ of GN20; Magorrian et al. 1998; Häring \& Rix 2004) by a factor of a few, but this offset is of the same order of magnitude as the uncertainties. In any case, these considerations show that the supermassive black hole properties implied by the above estimates appear to be reasonable. Assuming a canonical mass accretion efficiency of $\eta=0.1$, this also corresponds to an accretion rate of $\dot{M} \simeq 4-7 M_{\odot} \mathrm{yr}^{-1}$, or $\sim 0.2 \%-0.4 \%$ of the SFR. Given the underlying corrections and uncertainties, we consider these (plausible) values to be reliable to within a factor of three to five at best. 


\section{REFERENCES}

Alexander, D. M., Bauer, F. E., Brandt, W. N., et al. 2003, AJ, 126, 539

Alexander, D. M., Bauer, F. E., Chapman, S. C., et al. 2005a, ApJ, 632,736

Alexander, D. M., Brandt, W. N., Smail, I., et al. 2008, AJ, 135, 1968

Alexander, D. M., Smail, I., Bauer, F. E., et al. 2005b, Natur, 434, 738

Armus, L., Charmandaris, V., Bernard-Salas, J., et al. 2007, ApJ, 656, 148

Blain, A. W., Smail, I., Ivison, R. J., Kneib, J.-P., \& Frayer, D. T. 2002, PhR, 369,111

Brandl, B. R., Bernard-Salas, J., Spoon, H. W. W., et al. 2006, ApJ, 653, 1129

Capak, P., Carilli, C. L., Lee, N., et al. 2008, ApJL, 681, L53

Capak, P. L., Riechers, D., Scoville, N. Z., et al. 2011, Natur, 470, 233

Carilli, C. L., Daddi, E., Riechers, D., et al. 2010, ApJ, 714, 1407

Chabrier, G. 2003, PASP, 115, 763

Coppin, K., Pope, A., Menéndez-Delmestre, K., et al. 2010, ApJ, 713, 503

Coppin, K. E. K., Smail, I., Alexander, D. M., et al. 2009, MNRAS, 395, 1905

Daddi, E., Dannerbauer, H., Krips, M., et al. 2009b, ApJL, 695, L176

Daddi, E., Dannerbauer, H., Stern, D., et al. 2009a, ApJ, 694, 1517

Daddi, E., Dickinson, M., Morrison, G., et al. 2007, ApJ, 670, 156

Draine, B. T. 2003, ARA\&A, 41, 241

Draine, B. T., \& Li, A. 2007, ApJ, 657, 810

Farrah, D., Bernard-Salas, J., Spoon, H. W. W., et al. 2007, ApJ, 667, 149

Förster Schreiber, N. M., Sauvage, M., Charmandaris, V., et al. 2003, A\&A, 399,833

Genzel, R., Lutz, D., Sturm, E., et al. 1998, ApJ, 498, 579

Greve, T. R., Bertoldi, F., Smail, I., et al. 2005, MNRAS, 359, 1165

Häring, N., \& Rix, H.-W. 2004, ApJL, 604, L89

Helou, G., Soifer, B. T., \& Rowan-Robinson, M. 1985, ApJ, 298, L7

Hodge, J. A., Carilli, C. L., Walter, F., Daddi, E., \& Riechers, D. 2013, ApJ, 776,22

Hodge, J. A., Carilli, C. L., Walter, F., et al. 2012, ApJ, 760, 11

Houck, J. R., Roellig, T. L., van Cleve, J., et al. 2004, ApJS, 154, 18

Ivison, R. J., Magnelli, B., Ibar, E., et al. 2010, A\&A, 518, L31

Ivison, R. J., Papadopoulos, P. P., Smail, I., et al. 2011, MNRAS, 412, 1913

Iono, D., Peck, A. B., Pope, A., et al. 2006, ApJ, 640, L1

Laird, E. S., Nandra, K., Pope, A., \& Scott, D. 2010, MNRAS, 401, 2763
Lutz, D., Maiolino, R., Spoon, H. W. W., \& Moorwood, A. F. M. 2004, A\&A, 418, 465

Magdis, G. E., Daddi, E., Elbaz, D., et al. 2011, ApJL, 740, L15

Magorrian, J., Tremaine, S., Richstone, D., et al. 1998, AJ, 115, 2285

Menéndez-Delmestre, K., Blain, A. W., Alexander, D. M., et al. 2007, ApJL, 655, L65

Menéndez-Delmestre, K., Blain, A. W., Smail, I., et al. 2009, ApJ, 699, 667

Morrison, G. E., Owen, F. N., Dickinson, M., Ivison, R. J., \& Ibar, E. 2010, ApJS, 188,178

Nguyen, H. T., Schulz, B., Levenson, L., et al. 2010, A\&A, 518, L5

Peeters, E., Spoon, H. W. W., \& Tielens, A. G. G. M. 2004, ApJ, 613, 986

Petric, A. O., Armus, L., Howell, J., et al. 2011, ApJ, 730, 28

Pope, A., Borys, C., Scott, D., et al. 2005, MNRAS, 358, 149

Pope, A., Scott, D., Dickinson, M., et al. 2006, MNRAS, 370, 1185

Pope, A., Chary, R.-R., Alexander, D. M., et al. 2008, ApJ, 675, 1171

Renzini, A. 2006, ARA\&A, 44, 141

Riechers, D. A., Bradford, C. M., Clements, D. L., et al. 2013, Natur, 496, 329

Riechers, D. A., Capak, P. L., Carilli, C. L., et al. 2010, ApJL, 720, L131

Riechers, D. A., Carilli, C. L., Walter, F., et al. 2011a, ApJL, 733, L11

Riechers, D. A., Hodge, J., Walter, F., Carilli, C. L., \& Bertoldi, F. 2011b, ApJL, 739, L31

Riechers, D. A., Walter, F., Carilli, C. L., Bertoldi, F., \& Momjian, E. 2008a, ApJL, 686, L9

Riechers, D. A., Walter, F., Brewer, B. J., et al. 2008b, ApJ, 686, 851

Rigopoulou, D., Spoon, H. W. W., Genzel, R., et al. 1999, AJ, 118, 2625

Sajina, A., Yan, L., Armus, L., et al. 2007, ApJ, 664, 713

Spergel, D. N., Verde, L., Peiris, H. V., et al. 2003, ApJS, 148, 175

Spergel, D. N., Bean, R., Doré, O., et al. 2007, ApJS, 170, 377

Spoon, H. W. W., Armus, L., Cami, J., et al. 2004, ApJS, 154, 184

Spoon, H. W. W., Keane, J. V., Cami, J., et al. 2005, in IAU Symp. 231, Astrochemistry: Recent Successes and Current Challenges, ed. D. C. Lis, G. A. Blake, \& E. Herbst (Cambridge: Cambridge Univ. Press), 281

Tacconi, L. J., Genzel, R., Smail, I., et al. 2008, ApJ, 680, 246

Tran, Q. D., Lutz, D., Genzel, R., et al. 2001, ApJ, 552, 527

Vasudevan, R. V., \& Fabian, A. C. 2007, MNRAS, 381, 1235

Veilleux, S., Rupke, D. S. N., Kim, D.-C., et al. 2009, ApJS, 182, 628

Younger, J. D., Fazio, G. G., Wilner, D. J., et al. 2008, ApJ, 688, 59

Yun, M. S., Reddy, N. A., \& Condon, J. J. 2001, ApJ, 554, 803 